\title{
Nuclear magnetic resonance metabolic fingerprint of bevacizumab in mutant IDH1 glioma cells
}

\author{
Tanja Mesti ${ }^{1,6}$, Nadia Bouchemal ${ }^{2}$, Claire Banissi ${ }^{1}$, Mohamed N. Triba $^{2}$, \\ Carole Marbeuf-Gueye², Maja Cemazar ${ }^{3}$, Laurence Le Moyec ${ }^{4}$, Antoine F. Carpentier 1, 5, \\ Philippe Savarin ${ }^{3}$, Janja Ocvirk6
}

\author{
${ }^{1}$ Laboratoire de Recherches Biochirurgicales, Université Paris Descartes, Hôpital Européen Georges Pompidou, Paris, France \\ ${ }^{2}$ CSPBAT, UMR 7244, CNRS, Université Paris 13, Sorbonne Paris Cité, Bobigny, France \\ ${ }^{3}$ Department of Experimental Oncology, Institute of Oncology Ljubljana, Ljubljana, Slovenia \\ ${ }^{4}$ Unité de Biologie Intégrative des Adaptations à l'Exercice, Université d'Evry, Evry, France \\ ${ }^{5}$ Assistance Publique-Hôpitaux de Paris, Hôpital Avicenne, Service de Neurologie, Bobigny, France \\ ${ }^{6}$ Division of Medical Oncology, Institute of Oncology Ljubljana, Ljubljana, Slovenia
}

Radiol Oncol 2018; 52(4): 392-398.

Received 30 November 2017

Accepted 21 October 2018

Correspondence to: Assoc. Prof. Janja Ocvirk, M.D., Ph.D., Institute of Oncology Ljubljana, Zaloška 2, Ljubljana. Phone: +386 15879220 ; Fax: +386 15879 305; E-mail: jocvirk@onko-i.si

Disclosure: No potential conflicts of interest were disclosed.

\begin{abstract}
Background. Malignant gliomas are rapidly growing tumours that extensively invade the brain and have bad prognosis. Our study was performed to assess the metabolic effects of bevacizumab on the glioma cells carrying the IDHI mutation, a mutation, associated with better prognosis and treatment outcome. Bevacizumab is known to inhibit tumour growth by neutralizing the biological activity of vascular endothelial growth factor (VEGF). However, the direct effects of bevacizumab on tumour cells metabolism remain poorly known.

Materials and methods. The immunoassay and MTT assay were used to assess the concentration of secreted VEGF and cell viability after bevacizumab exposure. Metabolomic studies on cells were performed using high resolution magic angle spinning spectroscopy (HRMAS).

Results. mIDH1-U87 cells secreted VEGF (13 ng/mL). Regardless, bevacizumab had no cytotoxic effect, even after a $72 \mathrm{~h}$ exposure and with doses as high as $1 \mathrm{mg} / \mathrm{mL}$. Yet, HRMAS analysis showed a significant effect of bevacizumab $(0.1 \mathrm{mg} / \mathrm{mL})$ on the metabolic phenotype of $\mathrm{mIDH1}-\mathrm{U} 87$ cells with elevation of 2-hydroxyglutarate and changes in glutamine group metabolites (alanine, glutamate, glycine) and lipids (polyunsaturated fatty acids [PUFA], glycerophosphocholine, and phosphocholine).

Conclusions. In mIDHI-U87 cells, changes in glutamine group metabolites and lipids were identified as metabolic markers of bevacizumab treatment. These data support the possibility of a functional tricarboxylic acid cycle that runs in reductive manner, as a probable mechanism of action of bevacizumab in IDHI mutated gliomas and propose a new target pathway for effective treatment of malignant gliomas.
\end{abstract}

Key words: idh1 mutation, malignant glioma, bevacizumab, metabolic fingerprint

\section{Introduction}

Malignant gliomas are rapidly growing tumours that extensively invade the brain and critically depend upon angiogenesis. Malignant gliomas are known to secrete vascular endothelial growth fac- tor (VEGF) to stimulate angiogenesis. ${ }^{1}$ The IDH1 (NADP+-dependent isocitrate dehydrogenase) mutation occurs in the vast majority of WHO grade II or III gliomas and secondary glioblastomas. The p.Arg132His mutation (substitution from arginine to histidine) of isocitrate dehydrogenase 1 
(IDH1-R132H) is not only a frequent alteration (> $70 \%$ ) but is also a major prognostic marker in malignant gliomas. Patients with an IDH1 mutation have better treatment outcomes and better survival. ${ }^{2}$ A number of studies have analysed the role of IDH mutations in cancer. ${ }^{3,4}$ The main biochemical alteration associated with these mutations is the gain of a new enzymatic activity in which mutant IDH reduces $\alpha$-ketoglutarate $(\alpha-K G$, also called 2-oxoglutarate) to 2-hydroxyglutarate (2-HG). This is in contrast to wild-type IDH1, which catalyzes the NADP+-dependent oxidative decarboxylation of isocitrate into $\alpha$-KG. As a result, mutant IDH1 leads to elevated levels of $2-\mathrm{HG}$ in tumour cells. ${ }^{5}$ The tumorigenic effects of elevated 2-HG may be related to widespread changes in histone and DNA methylation. ${ }^{6}$

The use of metabolic profiling in conjunction with data mining tools allows the study of differences between normal and cancer cells and provides insights into the metabolic processes within cells and tumour tissues. NMR high resonance magic angle spinning (HRMAS) analysis is a very specific method with high potential for assessing subtle changes in the metabolic profiles of cells.-11 Using HRMAS, we have already shown that the VEGF inhibitor bevacizumab, which is used in recurrent glioblastoma, does not significantly impact the metabolism of wild-type U87 cells. This was in contrast to the VEGFR2-selective inhibitor SU1498 which elicits a dramatic increase in lipids, polyunsaturated fatty acids (PUFAs) in particular, due to an apoptotic response that is accompanied by accumulation of lipid droplets prior to DNA fragmentation, as we documented. ${ }^{12}$

To date, there has been no published study in the literature examining the direct metabolic effect of bevacizumab on mIDH1 cells. Therefore, we performed HRMAS analysis of the metabolic effect of bevacizumab $(0.1 \mathrm{mg} / \mathrm{mL})$ on a glioma U87 cell line that carries the IDH1-R132H mutation (mIDH1-U87) and we present our data. First, we assessed the drug's effects on cell proliferation, cell morphology, and VEGF secretion. Afterwards, we investigated the metabolic impact of bevacizumab on tumour cells by HRMAS. We report that bevacizumab has a significant effect on the metabolic phenotype of mIDH1-U87 cells, causing elevation of 2-hydroxyglutarate and changes in levels of the glutamine group of metabolites (alanine, glutamate, glycine) and in lipid signal (PUFA, glycerophosphocholine [GPC], and phosphocholine $[P C])$. Studying these metabolites by magnetic resonance spectroscopy (MRS) in patient samples could provide an early surrogate marker of bevacizumab response in tumour cells and, thus, might have a significant impact on clinical practice. These data support the possibility of a functional tricarboxylic acid cycle that runs in reductive manner, as a probable mechanism of action of bevacizumab in IDH1 mutated gliomas. With this we give not only new data of bevacizumab mechanism of action, but also we propose a new target pathway for effective treatment of malignant gliomas.

\section{Materials and methods}

\section{Cell phenotype, VEGF secretion and sensitivity to drugs}

The mIDH1-U87 (U87 cell line stably transfected with IDH1-R132H with a lentiviral vector, and was kindly provided by Marc Sanson [Hôpital de la Salpêtrière, Paris, France]) was maintained in Eagle's minimal essential medium (EMEM) with $10 \%$ foetal calf serum, $2 \mathrm{mM}$ L-glutamine, $100 \mathrm{U} /$ $\mathrm{mL}$ penicillin, and $100 \mu \mathrm{g} / \mathrm{mL}$ streptomycin (Lonza, Verviers, Belgium). bevacizumab (Roche, Paris, France) was diluted with culture medium to working concentrations before use. As a control, a stock solution containing the corresponding excipient was prepared with $60 \mathrm{mg} / \mathrm{mL}$ of trehalose dehydrate, $5.8 \mathrm{mg} / \mathrm{mL}$ sodium dihydrogen phosphate monohydrate, and $1.5 \mathrm{mg}$ di-sodium hydrogen phosphate dihydrate (all from Sigma Aldrich, SaintQuentin Fallavier, France). VEGF secretion was assessed with the Quantikine ELISA kit for human VEGF (R\&D Systems, Abingdon, UK), following the manufacturer's instructions. The cells proliferation was assessed as follows: The mIDH1-U87 cells were seeded into and allowed to attach overnight. Cells (seeded in 24-well plates; 30,000 cells/well) were treated for $72 \mathrm{~h}$ with bevacizumab (from 0.1 $\mathrm{mg} / \mathrm{mL}$ to $1 \mathrm{mg} / \mathrm{mL}$ ) in triplicate wells. Duplicate experiments were performed. Cell viability was then assessed with the MTT assay following the procedure of Mosmann..$^{13}$ The percentage of surviving cells is expressed as the ratio of optical density of treated cells versus untreated cells.

\section{${ }^{1} \mathrm{H}-\mathrm{NMR}$ spectroscopy}

Subconfluent mIDH1-U87 cells $\left(4 \times 10^{6}\right.$ cells $/ 60 \mathrm{~mm}$ diameter cell culture dishes) were incubated for $24 \mathrm{~h}$ and then treated with bevacizumab $(0.1 \mathrm{mg} /$ $\mathrm{mL})$ or trehalose $(0.24 \mathrm{mg} / \mathrm{mL})$ for $24 \mathrm{~h}$. The cells $\left(6 \times 10^{6}\right.$ cells/dish) were then harvested in $0.5 \mathrm{~mL}$ cold $\left(4^{\circ} \mathrm{C}\right)$ phosphate-buffered saline (PBS) in deu- 

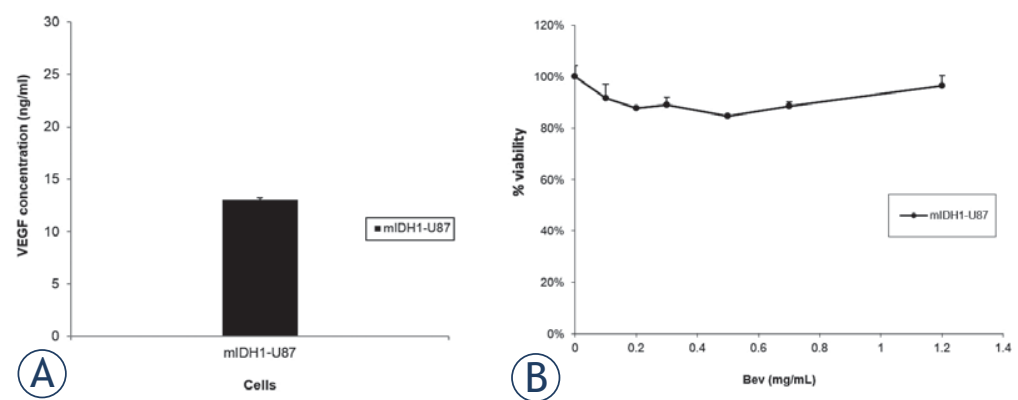

FIGURE 1. MIDH1-U87 vascular endothelial growth factor (VEGF) secretion and cell viability. (A) VEGF secretion ofmIDHI-U87 cells was assessed with immunoassay (B) Cell viability (\%) of mIDH1-U87 cell lines after $72 \mathrm{~h}$ bevacizumab treatment at different cell line viability. Concentrations $(0.1 \mathrm{mg} / \mathrm{mL}$ to $1 \mathrm{mg} / \mathrm{mL})$ was assessed with the MTT assay. Bevacizumab has no significant effect $(p>0.05)$ on $\mathrm{mIDHI}$-U87 cell line viability.

\section{Statistical analyses and metabolite identification in HRMAS spectra}

Data for physiological processes are shown as mean values of at least two different experiments and expressed as means \pm S.D. The data were first tested for normality of distribution and the differences between the experimental groups were evaluated by Student's t-test for one-way analysis of variance. A p value of less than $0.05(p<0.05)$ was considered to be statistically significant. SigmaPlot statistical software was used.

Principal component analysis (PCA) was conducted to detect any outliers based on NMR signal variability, defined as observations situated outside the $95 \%$ confidence region of the model. Orthogonal projection to latent structure (OPLS) analysis was performed when no significant differences were observed with the PCA analysis. A leave-one-out internal cross-validation procedure was used to calculate the predictability of the model. The ability of the model to describe data and to correctly predict new data is expressed by the values of the parameters R2 and Q2. R2=1 indicates a perfect description of the data while Q2 $=1$ indicates a perfect prediction of new data. Results are visualized by the scores and loadings plots. The scores plot shows the separation between groups. Scores are represented as a projection of the different samples on the predictive (Tpred) and the orthogonal (Torth) component. The loadings plot shows the distribution of the corresponding variables responsible for the separation observed in the scores plot. PCA and OPLS were conducted using SIMCA-P12 (Umetrics) and in-house Matlab (Mathworks) code based on the Trygg and Wold method. ${ }^{15}$ For metabolites considered as discriminant by the multivariate analysis, concentrations of metabolites were calculated by integration of their NMR signal.

\section{Results}

VEGF secretion was found $(13 \mathrm{ng} / \mathrm{mL})$ in the culture medium after $24 \mathrm{~h}$ incubation of mIDH1U87cells (Figure 1A). Bevacizumab, even in concentrations up to $1 \mathrm{mg} / \mathrm{mL}$, and regardless of the exposure time, had no effect on mIDH1-U87 cell line viability (Figure 1B).

In order to study the metabolic impact of bevacizumab on the mIDH1-U87 glioblastoma cell line, we characterized the bevacizumab fingerprint on mIDH1-U87 cells using the same approach as de- 
scribed for assessment of the metabolic fingerprint of bevacizumab on U87 cells, using trehalose-treated mIDH1-U87 cells as a control. The model was first calculated using a test set composed of 2 different culture sets (biological replicates) of trehalosetreated cells (each composed of 10 culture flasks), and 2 different cultures of bevacizumab-treated cells (each composed of 10 culture flasks). The corresponding score plot is presented in Figure 2. An OPLS model was calculated to characterize the metabolomic effect of bevacizumab on mIDH1U87 cells (Figure 2A). The predictive ability of the model $\mathrm{Q}^{2}$ was 0.93 ). To test the predictive ability of the model, a validation set was used. A projection of new samples (new trehalose-treated culture sets versus new bevacizumab-treated culture cells) was done using the model calculated with the test set. The score plot for the validation set is shown in Figure 2B. The AUROC obtained for the validation set was 0.82 , which corresponds to good sen- sitivity and specificity of the prediction. The corresponding loadings coefficients plot is shown in Figure 2C. Using 2D experiments, Chenomx and HMDB database, the main metabolites, modified by bevacizumab treatment, were identified as (Figure 2D and Table 1), choline metabolite, GPC, in the area $3.65-3.71 \mathrm{ppm}$, with an increase of $35 \%$, taurine (3.3-3.42 ppm) with an increase of $30 \%$, alanine (1.475 ppm), glutamate (2.11/2.34/2.55/3.02 ppm) with an increase of $25 \%$, creatine in the area $2.95 \mathrm{ppm}$ and $3.02 \mathrm{ppm}$ with an increase of $15 \%$ and $25 \%$, respectively, and 2-HG (1.84/2.24/3 ppm) with an increase of $20 \%$. Also, there were significant metabolite changes in the $3.2 \mathrm{ppm}$ area identified as phosphocholine with an increase of $7 \%$, in the same area where the GPC/choline ratio was also increased by $9 \%$. Glycine was detected as increased in the $3.6 \mathrm{ppm}$ area, but as NMR peaks were superimposed, the quantification of this metabolite was not possible.
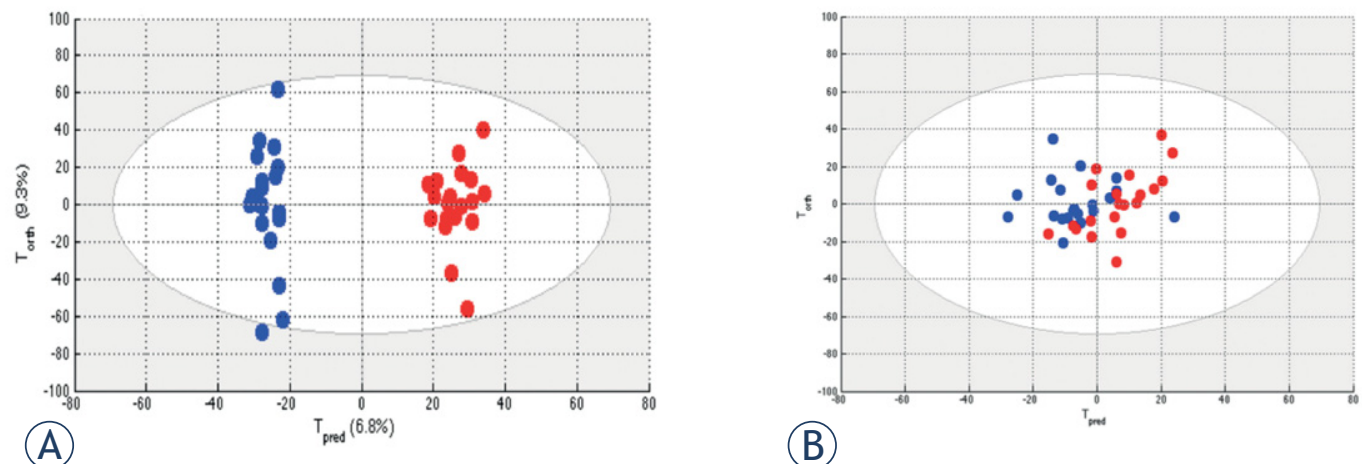

(B)

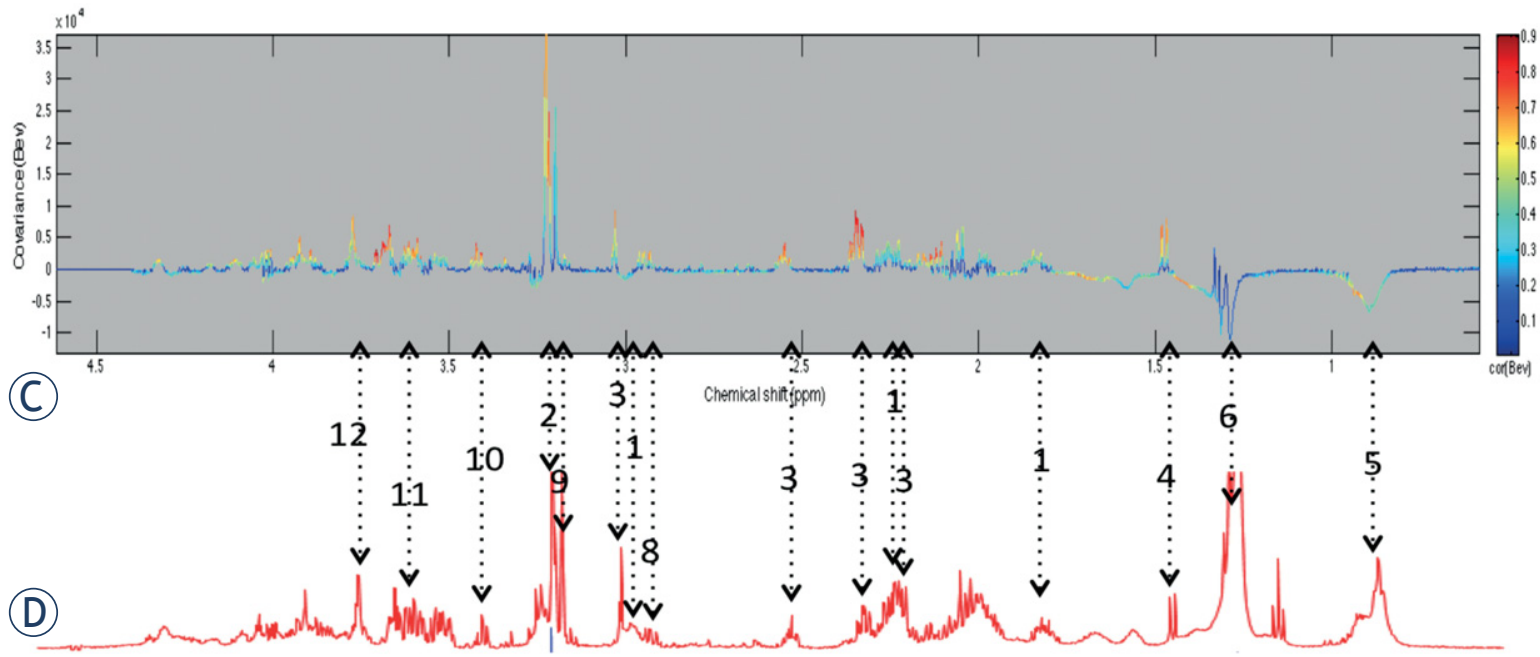

FIGURE 2. (A) Orthogonal projection to latent structure (OPLS)-DA score plot to discriminate metabolic effects of trehalose (blue) and bevacizumab (red) on mIDHI-U87 cells. (B) Projection of the spectra corresponding to the validation set. (C) OPLS loadings plot for mIDH1-U87 metabolic changes (significant changes marked with colours other than dark blue) after bevacizumab treatment. 'H CPMG spectrum of cells incubated with bevacizumab (D, RED). 


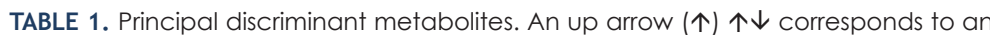
increase of the concentration induced by bevacizumab

\begin{tabular}{ccccc}
\hline $\begin{array}{c}\text { Peak } \\
\text { number }\end{array}$ & $\begin{array}{c}\text { Chemical shift } \\
(\text { ppm) }\end{array}$ & Attributions & $\begin{array}{c}\text { Concentration } \\
\text { variations Induced } \\
\text { by Bev }\end{array}$ \\
\hline 1 & $184 / 2.24 / 3$ & 2 OH-glutarate & $\uparrow$ & $20 \%$ \\
2 & 3.22 & GPC/chol & $\uparrow$ & $9 \%$ \\
3 & $2.11 / 2.34 / 2.55 / 302$ & glutamate & $\uparrow$ & $25 \%$ \\
4 & 1.475 & alanine & $\uparrow$ & $25 \%$ \\
5 & 0.88 & $(\mathrm{CH} 2)$ n-CH3 & $\downarrow$ & $32 \%$ \\
6 & 1.28 & $(\mathrm{CH}$ 2) -CH2-(CH2)m & $\downarrow$ & $20 \%$ \\
7 & 2.95 & creatine & $\uparrow$ & $15 \%$ \\
8 & 3.02 & creatine & $\uparrow$ & $25 \%$ \\
9 & 3.2 & Pcholine & $\uparrow$ & $7 \%$ \\
10 & $3.3-3.42$ & Taurine & $\uparrow$ & $30 \%$ \\
11 & 3.6 & glycine & $\uparrow$ & $\mathrm{ND}$ \\
12 & $3.65-3.71$ & GPC & $\uparrow$ & $35 \%$ \\
\hline
\end{tabular}

Bev = bevacizumab; $\mathrm{GPC}=$ glycerophosphocholine; $\mathrm{ND}=$ not determined; Pcholine $=$ phosphocholine of 2-HG in tumour cells. ${ }^{5}$ The exact mechanism through which mutant IDH1 and 2-HG induce oncogenesis continues to be investigated, but recent studies confirm the hypothesis that 2-HG can competitively inhibit $\alpha-K G$-dependent enzymes, which normally act to transfer amine groups from free amino acids to $\alpha-\mathrm{KG}$, as a first step in amino acid breakdown for oxidation in the tricarboxylic acid (TCA) cycle. ${ }^{6}$ TCA down-regulation has been associated with a selective advantage for cancer cells. Nutrients are converted to building blocks, such as amino acids and lipids, which are used for proliferation rather than being oxidized in the TCA cycle. ${ }^{16}$

According to our research data and using 2D experiments and the HMDB (Human Metabolome DataBase), the main metabolites modified by bevacizumab treatment were identified as GPC with a concentration increase of $35 \%$ in the area 3.65-3.71 ppm, taurine (3.3-3.42 ppm) with an increase of $30 \%$, and there were significant metabolite changes in the $3.2 \mathrm{ppm}$ area identified as PC with an increase of $7 \%$, and in the GPC/choline ratio with an increase of $9 \%$. GPC and PC have well-documented roles in membrane phospholipid breakdown ${ }^{17}$. Ex vivo MRS studies of brain tumours have identified several biomarkers of tumour growth and apoptosis, among which are increased levels of cholinecontaining compounds, possibly due to cell membrane disruption and altered phospholipid metabolism. ${ }^{18} \mathrm{PC}$ and GPC accumulation reflect early stages of growth arrest or apoptosis. ${ }^{19}$ The analysis of cisplatin-mediated apoptosis on ovarian cancer cells, showed enhanced apoptosis that was measured by increased PC and GPC concentrations. ${ }^{20}$ Elevated fatty acids and choline metabolism may be a sign of inhibited cell growth or apoptosis. In a study on human breast cancer cells, the increase in lipids (detected at 5.35, 1.3, and 0.9 ppm by NMR) during cytotoxic drug treatment was associated with mitochondrial damage, lipid droplet development, and formation of autophagic vacuoles. ${ }^{21}$ In malignant cells and tumours, appearance of a lipid peak at 1.3 ppm from $\mathrm{CH} 2 \mathrm{CH} 2 \mathrm{CH} 2$ has commonly been associated with ongoing cell death processes. ${ }^{22-24}$ In glioma cells, it has been demonstrated that PUFAs accumulate in BT4C glioma during gene therapy-induced programmed cell death (PCD), with pattern recognition identifying significant lipid changes 8-fold higher than normal in the area of $5.3 \mathrm{ppm}$ and $2.8 \mathrm{ppm}$, corresponding to $\mathrm{CH}=\mathrm{CH}$ and $\mathrm{CH}=\mathrm{CHCH}_{2} \mathrm{CH}=\mathrm{CH}$, and 2 fold higher than normal in the area of $0.9 \mathrm{ppm}$ and $1.3 \mathrm{ppm}$, corresponding to $\mathrm{CH}_{2}-\mathrm{CH}_{3}$ and $\mathrm{CH}_{2}-\mathrm{CH}_{2}-\mathrm{CH}_{2}$, as 
the most significant for monitoring the dynamics of PCD ${ }^{25}$ On the other hand, an HRMAS study on glioma biopsies has shown that the taurine signal may be a robust apoptotic biomarker that is independent of tumour necrotic status. Namely, this study showed that taurine significantly correlated with apoptosis in both non-necrotic $(\mathrm{R}=0.727, \mathrm{p}$ $=0.003)$ and necrotic $(\mathrm{R}=0.626, \mathrm{p}=0.0005)$ biopsies, but the PUFA changes in the $2.8 \mathrm{ppm}$ area, observed in other studies as a marker of apoptosis, correlated only in non-necrotic biopsies $(\mathrm{R}=0.705$, $p<0.005){ }^{26}$ This is in agreement with our data, as we identified significant changes in the lipid area (0.88 ppm and $1.28 \mathrm{ppm}$ ), with $\left(\mathrm{CH}_{2}\right) \mathrm{n}-\mathrm{CH}_{3}$ and $\left(\mathrm{CH}_{2}\right) \mathrm{n}-\mathrm{CH}_{2}-\left(\mathrm{CH}_{2}\right) \mathrm{m}$ signals, defined as PUFAs, being impacted with decreases of $32 \%$ and $20 \%$, respectively, and an increase of $30 \%$ of taurine (3.3 $3.42 \mathrm{ppm}$ ). This data also suggests that taurine may be a better biomarker of apoptosis in glial tumours than the change in PUFAs, which obviously differs according to the type of the tumour and correlates better with non-necrotic cancer types. Clearly it also correlates with the treatment used, as we had published the HRMAS analysis data of apoptotic impact of elevated PUFA by SU1498 treatment on U87 cells. ${ }^{12}$ The action of creatine has been shown to be linked with energy metabolism (formation of creatine phosphate) and inhibition of excitotoxicity. Decreased creatine has already been reported as a marker for highly proliferative cells and is found in gliomas. ${ }^{27}$ This is in contrast to its antioxidant and antiproliferative effects when elevated ${ }^{28}$, as also demonstrated in our work where bevacizumab treatment on mIDH1-U87 cells produced elevated creatine in the area $2.95 \mathrm{ppm}$ and $3.02 \mathrm{ppm}$ with increases of $15 \%$ and $25 \%$, respectively.

Furthermore, although we didn't detect lactate changes, other metabolites important for the TCA cycle and glutaminolysis were significantly impacted as shown by an increase of $20 \%$ for glutamate (2.11/2.34/2.55/3.02 ppm), alanine (1.475 ppm), and 2-HG (1.84/2.24/3 ppm), and a significant increase in glycine in the $3.6 \mathrm{ppm}$ area. As the NMR peaks were superimposed, the quantification of glycine was not possible. It has been already reported that mIDH1-U87 cells have lower concentrations of glutamate, lactate, and PC, and higher concentrations of GPC and 2-HG. ${ }^{29}$ Reitman et al., found similar changes in their analysis of IDH1-R132H mutated glioma cells, reporting a significant drop in glutamate, glutathione, aspartate, and $\mathrm{PC}$, and an increase in 2-HG and GPC. ${ }^{30}$ These data show that the TCA cycle is still functional with glutamine as a key nutrient providing substantial alternative in- put of nutrients ${ }^{31}$, and also proapoptotic activity, as glutamate, alanine, and glycine have already been reported as biomarker candidates for cell apoptosis. ${ }^{32}$ Metabolic rewiring is an established hallmark of cancer and, beyond the Warburg phenomenon with the use of glucose in hypoxic conditions and production of lactate, other changes can be observed, such as a functional TCA cycle that uses glutamine as a key nutrient to provide a substantial alternative input of nutrients into the TCA cycle (anaplerosis). ${ }^{31}$ A study on melanoma cells showed that in normoxia, but especially in hypoxia, glutamine provided a strong anaplerotic input to the TCA cycle via reductive flux from $\alpha-K G$ to citrate. $^{33}$ IDH1-R132H expression results in elevated flux from glutamine to 2-HG through glutamate and $\alpha-K G{ }^{34}$ Thus, glutamate may become depleted, as it is converted first to $\alpha-\mathrm{KG}$ and then to 2-HG. Except for the elevation of numerous free amino acids, IDH1-R132H cells show depletion of TCA cycle intermediates and elevation of lipid precursors such as GPC with a decrease of phosphatidylcholine.

The TCA cycle typically runs in the oxidative direction, but in our case, as other studies have shown, that in mammalian cells including glioblastoma cells, a reverse (reductive) flux between citrate and $\alpha$-ketoglutarate can also exist when glutamine is used as carbon source. ${ }^{35-37}$ In that manner, glutamine contributes to production of alanine, which is usually the product of glycolysis and fatty acid synthesis. In the process, free ammonia is generated that can induce autophagy. ${ }^{35-37}$

\section{Conclusions}

Our study was performed to assess the metabolic effects of Bevacizumab on the glioma cells carrying the IDH1 mutation, and for the first time we present data of the direct effects of bevacizumab on tumour cells metabolism. We observed dramatic changes in the metabolic phenotype of mIDH1-U87 cells after only $24 \mathrm{~h}$ exposure to Bevacizumab using HRMAS Proton Magnetic Resonance Spectroscopy Analysis. We detected elevation of 2-HG levels and changes in the area of glutaminolysis metabolites and lipids (PUFA, GPC, and PC) as early markers of the metabolic effect of bevacizumab on mIDH1U87 cells. These data support the possibility of a functional TCA cycle that runs in reductive direction inducing autophagy, as a probable mechanism of action of bevacizumab in IDH1-mutated glioma. Studying these metabolites by Magnetic Resonance 
Spectroscopy (MRS) in patient samples could provide an early surrogate marker of bevacizumab response in tumour cells and, thus, might have a significant impact on clinical practice.

With this we give not only new data of Bevacizumab mechanism of action, but also we propose a new target pathway for effective treatment of malignant gliomas.

\section{References}

1. Kaur B, Khwaja FW, Severson EA, Matheny SL, Brat DJ, Van Meir EG. Hypoxia and the hypoxia-inducible-factor pathway in glioma growth and angiogenesis. Neuro Oncol 2005; 7: 134-53. doi: 10.1215/S1152851704001115

2. Mesti T, Ocvirk J. Malignant gliomas: old and new systemic treatment approaches. Radiol Oncol 2016; 50: 129-38. doi: 10.1515/raon-2015-0003

3. Yang $\mathrm{H}$, Ye D, Guan $\mathrm{KL}$, Xiong $\mathrm{Y}$. IDH1 and IDH2 mutations in tumorigenesis: mechanistic insights and clinical perspectives. Clin Cancer Res 2012; 18: 5562-71. doi: 10.1158/1078-0432.CCR-12-1773

4. Losman JA, Kaelin WG, Jr. What a difference a hydroxyl makes: mutant IDH, (R)-2-hydroxyglutarate, and cancer. Genes Dev 2013; 27: 836-52. doi: 10.1101/gad.217406.113

5. Dang L, White DW, Gross S, Bennett BD, Bittinger MA, Driggers EM, et al. Cancer-associated IDH1 mutations produce 2-hydroxyglutarate. Nature 2009; 462: 739-44. doi: 10.1038/nature08617

6. Figueroa ME, Abdel-Wahab O, Lu C, Ward PS, Patel J, Shih A, et al. Leukemic IDH1 and IDH2 mutations result in a hypermethylation phenotype, disrupt TET2 function, and impair hematopoietic differentiation. Cancer Cell 2010 18: 553-67. doi: 10.1016/j.ccr.2010.11.015

7. Duarte IF, Marques J, Ladeirinha AF, Rocha C, Lamego I, Calheiros $R$, et al. Analytical approaches toward successful human cell metabolome studies by NMR spectroscopy. Anal Chem 2009; 81: 5023-32. doi: 10.1021/ac900545q

8. Cuperlovic-Culf M, Barnett DA, Culf AS, Chute I. Cell culture metabolomics: applications and future directions. Drug Discov Today 2010; 15: 610-21. doi: 10.1016/j.drudis.2010.06.012

9. Dietmair S, Timmins NE, Gray PP, Nielsen LK, Kromer JO. Towards quantitative metabolomics of mammalian cells: development of a metabolite extraction protocol. Anal Biochem 2010; 404: 155-64. doi: 10.1016/j. ab.2010.04.031

10. Kronthaler J, Gstraunthaler G, Heel C. Optimizing high-throughput metabolomic biomarker screening: a study of quenching solutions to freeze intracellular metabolism in CHO cells. Omics 2012; 16: 90-7. doi: 10.1089/ omi.2011.0048

11. Triba MN, Starzec A, Bouchemal N, Guenin E, Perret GY, Le Moyec L. Metabolomic profiling with NMR discriminates between biphosphonate and doxorubicin effects on B16 melanoma cells. NMR Biomed 2010; 23: 1009-16. doi: 10.1002/nbm.1516

12. Mesti T, Savarin P, Triba MN, Le Moyec L, Ocvirk J, Banissi C, et al. Metabolic impact of anti-angiogenic agents on U87 glioma cells. PLoS One 2014; 9: e99198 doi: 10.1371/journal.pone.0099198

13. Mosmann T. Rapid colorimetric assay for cellular growth and survival: application to proliferation and cytotoxicity assays. J Immunol Methods 1983; 65: 55-63. doi.org/10.1016/0022-1759(83)90303-4

14. Delaglio F, Grzesiek S, Vuister GW, Zhu G, Pfeifer J, Bax A. NMRPipe: a multidimensional spectral processing system based on UNIX pipes. J Biomol NMR 1995; 6: 277-93.

15. Trygg J, Wold S. Orthogonal projections to latent structures (O-PLS). Journa of Chemometrics 2002; 16: 119-28. doi: 10.1002/cem.695

16. Vander Heiden MG, Cantley LC, Thompson CB. Understanding the Warburg effect: The metabolic requirements of cell proliferation. Science 2009; 324 1029-33. doi: 10.1126/science.1160809

17. Billah MM, Anthes JC. The regulation and cellular functions of phosphatidylcholine hydrolysis. Biochem J 1990; 269: 281-91. PMCID: PMC1131573
18. Horska A, Barker PB. Imaging of brain tumors: MR spectroscopy and metabolic imaging. Neuroimaging Clin N Am 2010; 20: 293-310. doi: 10.1016/j. nic.2010.04.003

19. Cheng LL, Anthony DC, Comite AR, Black PM, Tzika AA, Gonzalez RG. Quantification of microheterogeneity in glioblastoma multiforme with ex vivo high-resolution magic-angle spinning (HRMAS) proton magnetic resonance spectroscopy. Neuro Oncol 2010; 2: 87-95. doi: 10.1093/neuonc/2.2.87

20. Maurmann L, Belkacemi L, Adams NR, Majmudar PM, Moghaddas S, Bose RN. A novel cisplatin mediated apoptosis pathway is associated with acid sphingomyelinase and FAS proapoptotic protein activation in ovarian cancer. Apoptosis 2015; 20: 960-74. doi: 10.1007/s10495-015-1124-2

21. Delikatny EJ, Cooper WA, Brammah S, Sathasivam N, Rideout DC. Nuclear magnetic resonance-visible lipids induced by cationic lipophilic chemotherapeutic agents are accompanied by increased lipid droplet formation and damaged mitochondria. Cancer Res 2002; 62: 1394-400.

22. Blankenberg FG, Storrs RW, Naumovski L, Goralski T, Spielman D. Detection of apoptotic cell death by proton nuclear magnetic resonance spectroscopy. Blood 1996; 87: 1951-6.

23. Blankenberg FG, Katsikis PD, Storrs RW, Beaulieu C, Spielman D, Chen JY, et al. Quantitative analysis of apoptotic cell death using proton nuclear magnetic resonance spectroscopy. Blood 1997; 89: 3778-86.

24. Al-Saffar NM, Titley JC, Robertson D, Clarke PA, Jackson LE, Leach MO, et al. Apoptosis is associated with triacylglycerol accumulation in Jurkat T-cells. $\mathrm{Br}$ J Cancer 2002; 86: 963-70. doi: 10.1038/sj.bjc.6600188.

25. Griffin JL, Lehtimäki KK, Valonen PK, Gröhn OH, Kettunen MI, Ylä-Herttuala $\mathrm{S}$, et al. Assignment of $1 \mathrm{H}$ nuclear magnetic resonance visible polyunsaturated fatty acids in BT4C gliomas undergoing ganciclovir-thymidine kinase gene therapy-induced programmed cell death. Cancer Res 2003; 63: 3195-201.

26. Opstad KS, Bell BA, Griffiths JR, Howe FA. Taurine: a potential marker of apoptosis in gliomas. Briti J Cancer 2009; 100: 789-94. doi: 10.1038/ sj.bjc.6604933

27. Tien RD, Lai PH, Smith JS, Lazeyras F. Single-voxel proton brain spectroscopy exam (PROBE/SV) in patients with primary brain tumors. AJR Am Roentgenol 1996; 167: 201-9. doi: 10.2214/ajr.167.1.8659372

28. Kolpakova ME, Veselkina OS, Vlasov TD. Creatine in cell metabolism and its protective action in cerebral ischemia. Neurosci Behav Physiol 2015; 45: 476-82. doi: 10.1007/s11055-015-0098-4

29. Izquierdo-Garcia JL, Viswanath P, Eriksson P, Chaumeil MM, Pieper RO, Phillips JJ, et al. Metabolic reprogramming in mutant IDH1 glioma cells. PLoS One 2015; 10: e0118781. doi: 10.1371/journal.pone.0118781

30. Reitman Z, Jin G, Karoly ED, Spasojevic I, Yang J, Kinzler KW, et al. Profiling the effects of isocitrate dehydrogenase 1 and 2 mutations on the cellular metabolome. Proc Natl Acad Sci USA 2011; 108: 3270-5. doi: 10.1073/ pnas.1019393108

31. Dang CV. Rethinking the Warburg effect with Myc micromanaging glutamine metabolism. Cancer Res 2010; 70: 859-62. doi: 10.1158/0008-5472. CAN-09-3556

32. Halama A, Moller G, Adamski J. Metabolic signatures in apoptotic human cancer cell lines. Omics 2011; 15: 325-35 doi: 10.1089/omi.2010.0121

33. Scott DA, Richardson AD, Filipp FV, Knutzen CA, Chiang GG, Ronai ZA, et al. Comparative metabolic flux profiling of melanoma cell lines: beyond the Warburg effect. J Biol Chem 2011; 286: 42626-34. doi: 10.1074/jbc. M111.282046

34. Dang L, White DW, Gross S, Bennett BD, Bittinger MA, Driggers EM, et al. Cancer-associated IDH1 mutations produce 2-hydroxyglutarate. Nature 2009; 462: 739-44. doi: 10.1038/nature08617

35. DeBerardinis RJ, Mancuso A, Daikhin E, Nissim I, Yudkoff M, Wehrli S, et al. Beyond aerobic glycolysis: transformed cells can engage in glutamine metabolism that exceeds the requirement for protein and nucleotide synthesis. Proc Natl Acad Sci USA 2007; 104: 19345-50. doi: 10.1073/ pnas.0709747104

36. Metallo CM, Walther JL, Stephanopoulos G. Evaluation of $13 \mathrm{C}$ isotopic tracers for metabolic flux analysis in mammalian cells. J Biotechnol 2009; 144: 167-74. doi: 10.1016/j.jbiotec.2009.07.010

37. Ward PS, Patel J, Wise DR, Abdel-Wahab O, Bennett BD, Coller HA, et al. The common feature of leukemia-associated IDH1 and IDH2 mutations is a neomorphic enzyme activity converting alpha-ketoglutarate to 2-hydroxyglutarate. Cancer Cell 2010; 17: 225-34. doi: 10.1016/j.ccr.2010.01.020 